\title{
Terahertz Dynamics of Excitons in GaAs/AlGaAs Quantum Wells
}

\author{
J. Černe, ${ }^{1}$ J. Kono, ${ }^{2, *}$ M. S. Sherwin, ${ }^{1}$ M. Sundaram, ${ }^{3, \dagger}$ A. C. Gossard ${ }^{3}$ and G. E. W. Bauer ${ }^{4}$ \\ ${ }^{1}$ Department of Physics and Center for Free-Electron Laser Studies, University of California, Santa Barbara, California 93106 \\ ${ }^{2}$ Quantum Transition Project, Research Development Corporation of Japan, Tokyo 153, Japan \\ ${ }^{3}$ Materials Department, University of California, Santa Barbara, California 93106 \\ ${ }^{4}$ Department of Applied Physics, Delft University of Technology, Lorentzweg 1, 2628 CJ Delft, The Netherlands
}

(Received 14 February 1996)

\begin{abstract}
By monitoring changes in excitonic photoluminescence that are induced by far-infrared (FIR) radiation, we observed resonant FIR absorption by magnetoexcitons in GaAs/AlGaAs quantum wells. The dominant resonance is assigned to the $1 s \rightarrow 2 p^{+}$transition of the heavy-hole exciton, and agrees well with theory. At low FIR and interband excitation intensities, the $1 s \rightarrow 2 p^{+}$absorption feature is very narrow and broadens as either of these intensities is increased. The $1 s \rightarrow 2 p^{+}$absorption feature persists even when the FIR electric field is comparable to the electric field which binds the exciton. [S0031-9007(96)00906-4]
\end{abstract}

PACS numbers: 73.20.Dx, 71.35.Ji, 78.66.Fd

Correlated electron-hole pairs form excitons in semiconductor heterostructures. Excitons in GaAs are hydrogenlike systems with Bohr radii of order $100 \AA$, and binding energies of order $10 \mathrm{meV}$. The importance and much of the rich structure of excitons have been revealed by extensive studies using one- and two-photon interband spectroscopies $(0.75-1.5 \mathrm{eV}$ in GaAs) [1]. However, very limited research has succeeded in directly exploring the internal dynamics of excitons [2-4]. In such studies, near-infrared (NIR) photons create excitons, and then far-infrared (FIR) radiation (of order $10 \mathrm{meV}, 2.4 \mathrm{THz}$ ) manipulates them. At low FIR intensities, one expects to observe directly transitions between even- and odd-parity states of the exciton, which are not observable with linear interband spectroscopy. Such transitions provide new, sensitive tests for the theory of excitons, which is fundamental in the physics of semiconductors. At higher FIR intensities, it is possible to reach a nonperturbative regime in which the energy associated with the FIR electric field coupling to the exciton is comparable to both the binding energy and the FIR photon energy.

Undoped direct (type I) quantum wells (QWs) are especially interesting since they are so commonly used and provide a simple model system for theoretical analysis. However, the short lifetime of excitons in type I QWs makes it difficult to achieve the large population of cold excitons required for FIR absorption studies. Recent experimental progress has been made in QWs using photoinduced absorption in staggered (type II) QWs [3] and time-resolved terahertz spectroscopy in type I QWs [4]. In this Letter, we have used optically detected FIR absorption to observe, for the first time, internal transitions of magnetoexcitons in type I QWs.

In optical detection of FIR resonance (ODR), one monitors changes in photoluminescence (PL) that result from FIR absorption. Previous experiments conducted in undoped QWs at zero magnetic field have shown that intense FIR radiation quenches the PL amplitude [5,6]. This PL quenching is consistent with the interpretation that the FIR radiation heats photoinjected electrons and holes, which in turn heat and decrease peak emission from luminescing excitons [6]. Numerous experiments have optically detected cyclotron resonance [7-13], and impurity transitions $[14,15]$ in various bulk semiconductors and quantum heterostructures. The equivalence of ODR and conventional transmission techniques in determining the frequency of absorption resonances has been shown clearly (for example, see $[10,11])$.

Several GaAs/AlGaAs undoped, molecular beam epitaxy-grown QW samples were investigated in this study. Sample 1 consisted of $50100 \AA$ wide GaAs QWs between $150 \AA$ thick $\mathrm{Al}_{0.3} \mathrm{Ga}_{0.7} \mathrm{As}$ barriers [5]. Sample 2 contained six single GaAs QWs with widths of 35,50 , 70, 100 and $140 \AA$. Finally, sample 3, consisting of a $150 \AA$ thick GaAs QW was examined. The typical background impurity concentration for all the samples is $10^{13}-10^{14} \mathrm{~cm}^{-3}$ and is mostly $p$ type.

UCSB's free-electron lasers (FEL) provide intense radiation (up to $1 \mathrm{MW} / \mathrm{cm}^{2}$ ) that can be continuously tuned from 4 to $160 \mathrm{~cm}^{-1}(0.5-20 \mathrm{meV}, 0.12-4.8 \mathrm{THz})$. As can be seen in the inset of Fig. 1(a), an $\mathrm{Ar}^{+}$laser was used to create electron-hole pairs in the undoped sample at $9 \mathrm{~K}$. Simultaneously, FIR radiation with the electric field polarized in the plane of the QW, and, therefore, not coupling to intersubband transitions, passed through the sample. Typical data were recorded with $\mathrm{Ar}^{+}$allline laser excitation intensity of $\approx 100 \mathrm{~W} \mathrm{~cm}^{-2}$ creating an exciton density of approximately $3 \times 10^{10} \mathrm{~cm}^{-2}$ per well in sample 1 [16]. The resulting PL was captured by $1850 \mu \mathrm{m}$ diameter optic fibers that surround a central excitation laser fiber. The PL was delivered to a monochromator and a cooled GaAs photomultiplier tube. The output of the $\mathrm{Ar}^{+}$laser was modulated acoustooptically to produce a $20 \mu$ s visible excitation pulse that coincided with the $5 \mu$ s FIR pulse at the sample. Since the FIR pulse is much longer than any carrier relaxation 


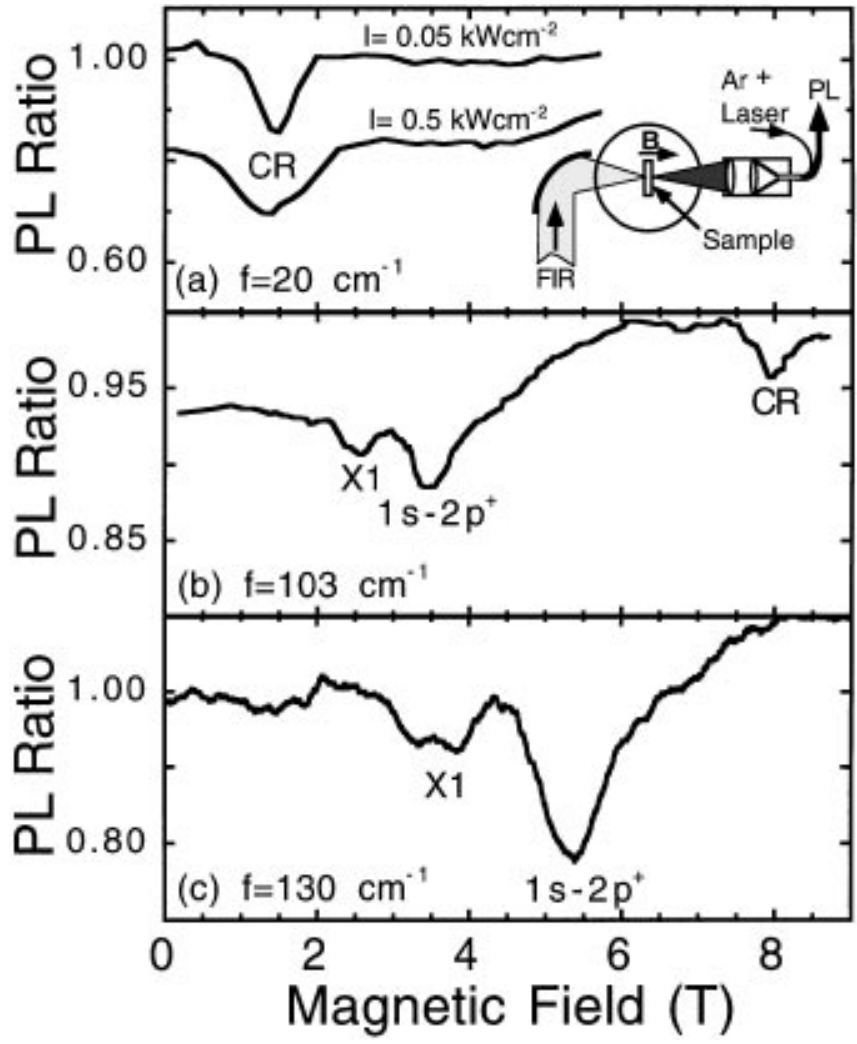

FIG. 1. The ratio of the PL amplitude with and without FIR irradiation as function of magnetic field for sample 1 at three FIR frequencies. The inset in (a) shows a schematic of the experimental setup.

time, the measurement is in steady state. The PL change during the FIR pulse was due to carrier heating; no lattice heating was observed [6]. The PL immediately after the FIR pulse was used to normalize all the data.

The monochromator was set to detect the peak of the PL from the $1 s$ heavy-hole free exciton. The magnetic field $B$ was varied to sweep FIR resonances through the fixed energy of the FIR radiation. At each $B$, the wavelength of the monochromator was adjusted to track the energy of the PL peak, which showed the expected diamagnetic shift. FIR intensity was measured absolutely as described in Ref. [6].

Figure 1 plots the ratio of the PL amplitudes with and without FIR irradiation as a function of $B$ for sample 1. A series of resonances is observed. We focus on two dominant resonances which are observed in all the samples that we studied. We assign these to FIRinduced electron cyclotron resonance (CR) [Figs. 1(a) and 1(b)] and $1 s \rightarrow 2 p^{+}$(as discussed below) excitonic transitions [Figs. 1(b) and 1(c)]. The weaker resonances (X1) at magnetic fields below the $1 s \rightarrow 2 p^{+}$transition are probably excitonic features as well (e.g., $1 s \rightarrow 3 p^{+}$), but were not observed in all samples. The FIR resonances were preserved when a Ti:sapphire laser excited carriers only into the QWs, below the barrier band gap.
In Fig. 2, the PL ratio as a function of $B$ is shown for several visible [Fig. 2(a)] and FIR [Fig. 2(b)] intensities in sample 1. The traces are not offset. Both the CR and excitonic transitions are visible. At the lowest FIR and visible intensities [top trace in Fig. 2(a)] the PL is enhanced at all magnetic fields except at $1.9 \mathrm{~T}$, where there is a sharp feature less than $0.3 \mathrm{~T}\left(4 \mathrm{~cm}^{-1}\right)$ wide. This feature is assigned to the $1 s \rightarrow 2 p^{+}$freeexciton resonance and broadens dramatically as the visible excitation intensity is increased. The baseline also falls below 1 and CR becomes observable at the higher visible excitation intensities. The dependence on visible excitation intensity is strongest at low FIR intensity. Figure 2(b) shows the dependence on FIR intensity for a fixed visible excitation intensity. Increasing the FIR intensity broadens the absorption features and lowers the PL ratio baseline. This means that higher FIR intensities produce significant off-resonance PL quenching at all magnetic fields and visible intensities.

The evolution of cyclotron and exciton energies as $B$ is increased in sample 1 is shown in Fig. 3. The solid symbols show the energies of the most prominent minima in the PL ratio as a function of $B$, whereas the empty circles represent the $1 s-2 s$ energy spacing of the heavy-hole exciton as deduced from interband PL and photoluminescence excitation (PLE) measurements. The cyclotron frequency is linear with $B$ and suggests an electronic effective mass of $0.073 m_{0}$, which is in agreement with theory [17] and experiment (for example, [13]) for a $100 \AA \mathrm{GaAs} \mathrm{QW}$. The slope for the strongest exciton transition (solid circles) is roughly the same as that for CR, indicating that this is a $1 s \rightarrow 2 p^{+}$. like transition in the low-field, hydrogenic notation [18].

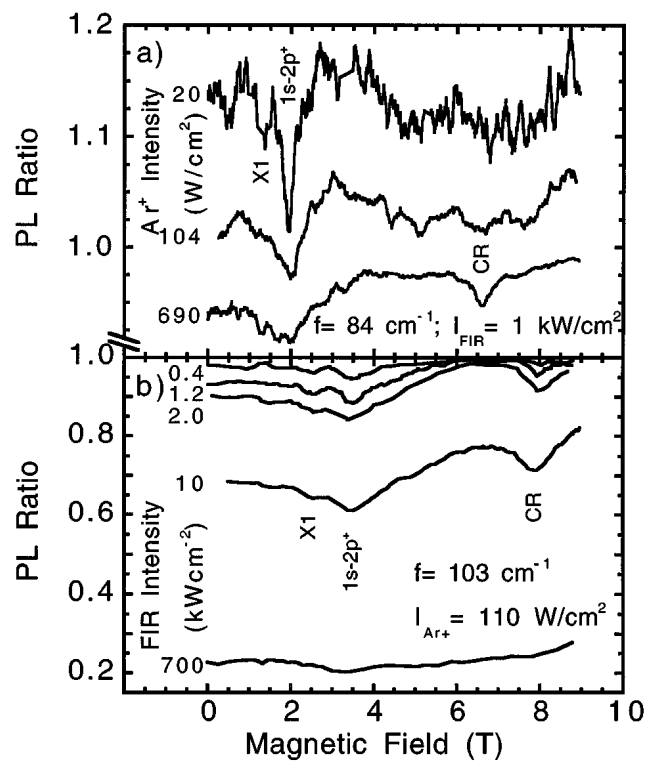

FIG. 2. The PL ratio as a function of magnetic field is shown for several visible (a) and FIR (b) intensities in sample 1. The traces are not offset. 


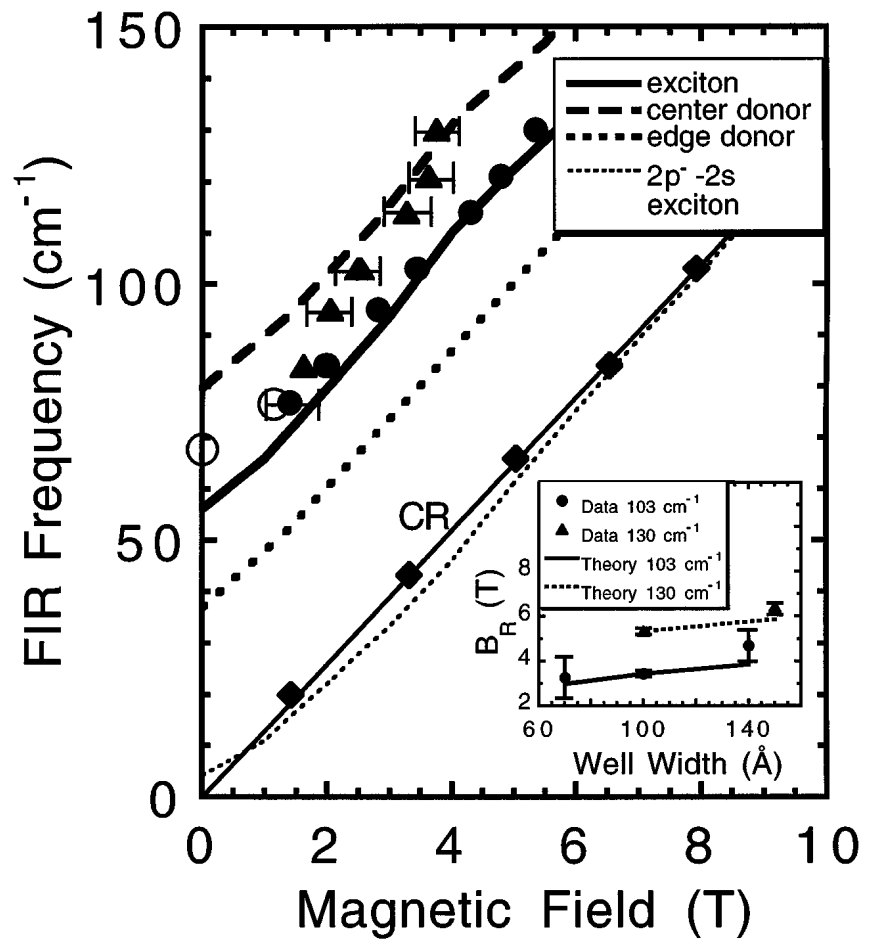

FIG. 3. The solid symbols show the energy of the various FIR resonances as a function of magnetic field in sample 1. The excitonic transitions such as the $1 s \rightarrow 2 p^{+}$(solid circles) and higher energy transitions (solid triangles) can be seen in addition to the free-electron cyclotron resonance (solid diamonds). The thick solid line and thin dotted line represent calculations using excitonic theory [20], while the dashed lines are calculated using donor theory [18]. The thin solid line is free-electron CR with an effective mass of $0.073 m_{0}$. The $1 s-2 s$ energy spacing of the heavy-hole exciton (large empty circles) was deduced from interband photoluminescence excitation measurements. The inset shows the magnetic field at which the $1 s \rightarrow 2 p^{+}$exciton transition occurs at two FIR frequencies in four QWs.

Weaker, higher energy transitions (solid triangles) have a clearly larger slope, indicating that they are transitions from the $1 s$ ground state to even higher energy states. The inset of Fig. 3 shows the magnetic field at which the $1 s \rightarrow 2 p^{+}$-like transition occurs in four QWs (70, 100,140 , and $150 \AA$ ) at two FIR frequencies (103 and $130 \mathrm{~cm}^{-1}$ ).

The $1 s \rightarrow 2 p^{+}$transition in the top trace of Fig. 2(a) is roughly 4 times narrower than the $1 s$ heavy-hole exciton PL line. This may be explained by the fact that the $1 s \rightarrow 2 p^{+}$transition energy depends only weakly on well width and hence is less sensitive to fluctuations in the well: $\partial E_{1 s \rightarrow 2 p^{+}} / \partial L_{z}=\left(\partial E_{1 s \rightarrow 2 p^{+}} / \partial B\right)\left(\partial B / \partial L_{z}\right)=$ $0.03 \mathrm{meV} / \AA$, where the derivatives on the right-hand side are calculated from experimental data of Fig. 3 and its inset, respectively. For comparison, the interband electronheavy-hole (e1-hh1) spacing is much more sensitive to the well width, varying like $1 \mathrm{meV} / \AA$ [19].

We compare our experimental results to calculations of FIR transitions in excitons and neutral donors. Bauer and
Ando [20] calculated the energy levels of magnetoexcitons in $100 \AA$ wide GaAs QWs within the framework of the effective mass approximation, taking into account the complexity of the valence band. The thick solid and the thin dotted lines in Fig. 3 show the calculated energies for the $1 s \rightarrow 2 p^{+}$and $2 p^{-} \rightarrow 2 s$ excitonic transitions, respectively. The lines in the inset represent calculated resonant magnetic fields for excitons in wells of differing widths. These calculations used a standard set of parameters which were not adjusted to fit the data [20].

The donor internal transition energies depend critically on the location of the donor in the QW. The binding energies and FIR transition energies for donors at different positions in GaAs QWs in the presence of magnetic fields have been calculated in detail by Greene and Lane [18]. This theory has been extensively verified by experiments [15,21]. The calculated $1 s \rightarrow 2 p^{+}$transition energies in Fig. 3 include donors that are centered in the QW and those that are located at the edge of the QW.

There is strong evidence to suggest that the $1 s \rightarrow 2 p^{+}$ data reported here are not consistent with FIR absorption by neutral donors (ODR signals due to very low concentrations of neutral donors have been recently observed in high-purity epitaxial GaAs [14]). First, the background impurity concentration $\left(10^{7}-10^{8} \mathrm{~cm}^{-2}\right)$ is 10 to 100 times smaller than the exciton sheet density $\left(10^{9}-5 \times 10^{10} \mathrm{~cm}^{-2}\right)$ [22]. Furthermore, most of these impurities are $p$ type, so even fewer donors are able to contribute to a $1 s \rightarrow 2 p^{+}$-like resonance. Finally, predictions from donor theory [18] and previous experimental measurements on donors [15,21] do not agree with our data, as can be seen in Fig. 3. For donors that are centered in the $100 \AA \mathrm{GaAs}$ well, the $1 s \rightarrow 2 p^{+}$transition energy is more than $1 \mathrm{meV}$ higher than the data, while for donors located at the edge of the $\mathrm{QW}$, the $1 s \rightarrow 2 p^{+}$ transition energy is more than $3 \mathrm{meV}$ lower than the data. The narrow $(0.3 \mathrm{~T})$ and symmetric line shape observed in our experiment [see Fig. 2(a)] is incompatible with the broad (up to $4 \mathrm{~T}$ ) and asymmetric line shape that would result from a uniform distribution of donors. The only possible donor configuration that is consistent with the observed line shape is having all the donors at the edge of the QW to form a narrow distribution of absorbers. The theoretical curve clearly shows that the $1 s \rightarrow 2 p^{+}$-like resonances are not from edge donors.

On the other hand, agreement with excitonic theory is good. The fact that these transitions were universally observed in all the samples suggests that these are intrinsically excitonic. The well-width dependence of the magnetic field required to bring the $1 s \rightarrow 2 p^{+}$transition into resonance with the FIR radiation agrees well with exciton theory, especially for the narrower QWs (see inset of Fig. 3). Finally, the $1 s-2 s$ energy separation obtained with PL and PLE agrees very well with the $1 s \rightarrow 2 p^{+}$results and with theory, which predicts a $1 s-2 s$ energy separation of $60.6 \mathrm{~cm}^{-1}$ at zero magnetic 
field. Since the $1 s-2 s$ spacing was obtained using excitonic PLE (detection was at the $1 s$ heavy-hole freeexciton peak), this energy spacing is clearly attributable to heavy-hole excitons and not due to neutral donors. The lowest frequency resonance, which agrees with CR, agrees almost as well with the predicted $2 p^{-} \rightarrow 2 s$ excitonic transition. We favor the assignment to $\mathrm{CR}$, but further studies are required to resolve this definitively.

We conclude by discussing the power dependence of the line shape of the ODR signal. At the lowest interband and FIR intensities in Fig. 2(a), we observed nonresonant PL enhancement. This is consistent with thermal ionization of impurity-bound excitons, which can then contribute to the free-exciton PL [15]. The sharp PL quenching observed at $1.9 \mathrm{~T}$ in the top spectrum of Fig. 2(a) is due to resonant FIR-induced photothermal ionization of free excitons. This result further rules out impurity absorption of FIR radiation, which would have led to heating and hence PL enhancement. At moderate visible intensities in Fig. 2(a), carriers are warmer and impurity-bound excitons have been ionized even in the absence of FIR radiation. For moderate FIR intensities [Fig. 2(b)], significant PL quenching occurs for all magnetic fields. A similar type of background signal due to hot free carriers has been seen in microwave ODCR experiments in bulk semiconductors [23]. At the highest FIR intensities ( $700 \mathrm{~kW} \mathrm{~cm}^{-2}$ inside the sample), the oscillating FIR electric field is $12 \mathrm{kV} / \mathrm{cm}$, which is comparable to the static electric field which binds the $1 s$ exciton $(10 \mathrm{meV} / 100 \AA)$. At such intensities, the unperturbed transition energies and eigenstates should not be relevant. It is thus somewhat surprising that the minimum in the PL ratio which we assign to the $1 s \rightarrow 2 p^{+}$ transition in Fig. 2(b) persists, without obvious shifts, to the highest FIR intensities. We are currently exploring the high-intensity effects in detail.

The authors gratefully acknowledge discussions with T. Inoshita, T. Ando, H. Sakaki, S. J. Allen, Jr., H. Akiyama, R. P. Mirin, R. Ram, A. Imamoglu, B. D. McCombe, and K. Unterrainer. R. P. Mirin and J. Ko also donated samples. We are grateful to A. Guitierrez for his work on the remote PL probe. We would also like to thank D. P. Enyeart, J. R. Allen, and J. P. Kaminski at the Center for Free-Electron Laser Studies for their technical support. This work has been supported by the NSF Science and Technology Center for Quantized Electronic Structures Grant No. DMR 91-20007, JRDC Quantum Transition Project, Grants No. ONR N00014-K-0692, and No. AFOSR F 49620-94-1-0158.
Note added. - Similar results have been obtained by Salib et al. [24].

*Visiting scientist at the Center for Free-Electron Laser Studies, University of California, Santa Barbara, CA 93106.

†Present address: JPL, NASA, MS 302-231, 4800 Oak Grove Drive, Pasadena, CA 91109.

[1] See, e.g., The Spectroscopy of Semiconductors, Semiconductors and Semimetals, Vol. 36, edited by D. G. Seiler and D. L. Littler (Academic Press, New York, 1992).

[2] Excitons in bulk germanium and silicon have been examined in the FIR by several experiments. See, e.g., E. M. Gershenzon et al., Sov. Phys. JETP 43, 116 (1976); D. Labrie et al., Phys. Rev. Lett. 61, 1882 (1988).

[3] C. C. Hodge et al., Phys. Rev. B 41, 12319 (1990).

[4] R. H. M. Groeneveld and D. Gryshkowsky, J. Opt. Soc. Am. 11, 2502 (1994).

[5] S. M. Quinlan et al., Phys. Rev. B 45, 9428 (1992).

[6] J. Černe et al., Phys. Rev. B 51, 5253 (1995).

[7] P. G. Baranov et al., JETP Lett. 26, 249 (1977).

[8] R. Romestain and C. Weisbuch, Phys. Rev. Lett. 45, 2067 (1980).

[9] M. G. Wright et al., Semicond. Sci. Technol. 5, 438 (1990).

[10] N. Ahmed et al., Semicond. Sci. Technol. 7, 357 (1992).

[11] S. I. Gubarev et al., JETP Lett. 54, 355 (1991).

[12] A. Moll et al., Phys. Rev. B 45, 1504 (1992).

[13] R. J. Warburton et al., Phys. Rev. B 46, 13394 (1992).

[14] J. G. Michels et al., Semicond. Sci. Technol. 9, 198 (1994).

[15] J. Kono et al., Phys. Rev. B 52, R8654 (1995).

[16] The exciton density was determined using the absorption coefficient for GaAs at $532 \mathrm{~nm}$ of $8 \times 10^{4} \mathrm{~cm}^{-1}$ and an exciton lifetime of $0.5 \mathrm{~ns}$.

[17] U. Ekenberg, Phys. Rev. B 40, 7714 (1989).

[18] R. L. Greene and P. Lane, Phys. Rev. B 34, 8639 (1986).

[19] J. Christen and D. Bimberg, Phys. Rev. B 42, 7213 (1990).

[20] G.E.W. Bauer and T. Ando, Phys. Rev. B 38, 6015 (1988). See also L. C. Andreani and S. Pasquarello, Phys. Rev. B 42, 8928 (1990).

[21] N. C. Jarosik et al., Phys. Rev. Lett. 54, 1283 (1985).

[22] Most other ODR experiments in QWs used typical excitation intensities below $2 \mathrm{~W} \mathrm{~cm}^{-2}$ [15] or $5 \mathrm{~mW} \mathrm{~cm}^{-2}$ $[13,14]$ compared to 5 to $160 \mathrm{~W} \mathrm{~cm}^{-2}$ used in this experiment. The lower excitation intensities translated in exciton densities that were less than or equal to the residual donor concentration $[10,14]$.

[23] F. P. Wang et al., Phys. Rev. B 39, 11195 (1989).

[24] S. Salib et al., Phys. Rev. Lett. 77, 1135 (1996). 